\title{
Stage IV Urethral Cancer AJCC v7
}

National Cancer Institute

\section{Source}

National Cancer Institute. Stage IV Urethral Cancer A/CC V7. NCI Thesaurus. Code C6200.

Stage IV includes: (T4, N0, M0); (T4, N1, M0); (Any T, N2, M0), (Any T, Any N, M1). T4:

Tumor invades other adjacent organs. N0: No regional lymph node metastasis. N1:

Metastasis in a single lymph node $2 \mathrm{~cm}$ or less in greatest dimension. N2: Metastasis in a single lymph node more than $2 \mathrm{~cm}$ in greatest dimension, or in multiple lymph nodes. M0: No distant metastasis. M1: Distant metastasis. (AJCC 7th ed.) 